\title{
Fast Arbitrary Waveform Generation by Using Digital Micro-Mirror Arrays
}

\author{
Salih K. Kalyoncu ${ }^{1}$, Yuewang Huang ${ }^{1}$, Qi Song ${ }^{1}$ and Ozdal Boyraz ${ }^{1,2}$ \\ ${ }^{1}$ EECS Department, University of California, Irvine, 92697, USA \\ ${ }^{2}$ EE Department, founding member, Istanbul Sehir University, Istanbul, Turkey \\ Email: oboyraz@uci.edu
}

\begin{abstract}
We demonstrate fast optical arbitrary waveform generation by using MEMS digital micro-mirror arrays. Experimentally, we obtain $\sim 120 \mathrm{MHz}$ waveforms that can be controlled by using $1024 \times 768$ mirror arrays. $1 \mathrm{GHz}$ waveforms reconfigurable in $1 \mu \mathrm{s}$ are also achievable.
\end{abstract}

\section{Introduction}

Arbitrary waveform generators (AWGs) are highly desired in many areas such as wideband communication systems, instrument diagnostics, remote sensing and radar applications. Optically assisted AWGs provide much better performance than the electronic AWGs in terms of the speed and the dynamic range resolution [1]. Space wavelength mapping followed by spatial light modulation has been recently used for waveform generator by shaping the spectrum of the broadband source with the spatial light modulator (SLM) [2-3]. Digital micro-mirror device (DMD) providing a high reflection, exceptional stability and excellent controllability over thousands of individual micro mirrors can be used as a digital spatial light modulator [4]. In this paper, we propose and demonstrate a novel method to generate optical arbitrary waveforms by using these MEMS based digital micro-mirror arrays. In this preliminary experimental work we demonstrate square and sawtooth waveforms at $\sim 120 \mathrm{MHz}$ by using $1024 \times 768$ mirror arrays. By using the state of the art MEMS technology arbitrary waveforms up to $1 \mathrm{GHz}$ rate with $1 \mu \mathrm{s}$ duration are achievable.

\section{Approach and Experimental Setup}

The experimental setup of optical AWG is illustrated in Fig 1a. The system is mainly designed by combining the broadband illumination, wavelength-time mapping, space-wavelength mapping, and spectral shaping modules. Supercontinuum (SC) pulses with $20 \mathrm{~nm}$ bandwidth centered at $1590 \mathrm{~nm}$ are used as a broadband illumination and propagated through the dispersion compensation module (DCM with $-675 \mathrm{ps} / \mathrm{nm}$ ) to map the wavelength information into temporal waveform for real time detection. In order to compensate the system losses and to obtain a better signal to noise ratio (SNR), a flat gain Raman amplifier ( $10 \mathrm{~dB}$ net gain and $<0.5 \mathrm{~dB}$ gain ripple) is designed to provide a uniform amplification through the DCM. The supercontinuum pulses are spatially dispersed by the diffractive optics (diffraction grating with $6001 \mathrm{ines} / \mathrm{mm}$ ) and sent on to the DMD chipset. The DMD chipset consists of $1024 \times 768$ individually controllable micro mirrors with $10.8 \mu \mathrm{m}$ size. Each mirror can be assigned to either ON $\left(+12^{\circ}\right)$ or OFF $\left(-12^{\circ}\right)$ states to direct the light. The detector is placed through the ON direction.
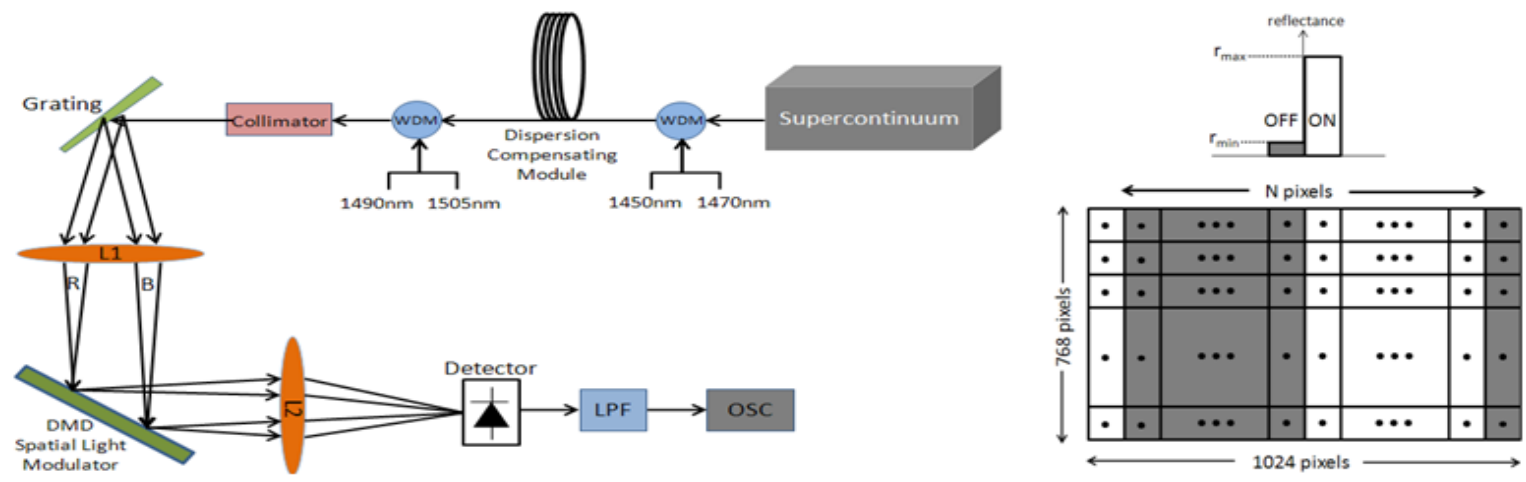

Fig 1. The experimental setup (1a) for the all-optical arbitrary waveform generator (AWG). The position of the grating, first lens (L1) and the DMD are set for maximum resolution and zero net dispersion [5]. The DMD chipset (1b) and the implemented modulation pattern [4].

The spectrum of the pulses is spatially modulated by the user-defined pattern of individual micro-mirrors shown in Fig 1b. Since the pulses are temporally dispersed by the DCM, the spatial modulation due to DMD mirrors are 
mapped into the temporal waveform and can be easily captured by a single photo detector $(>1.2 \mathrm{GHz})$ and oscilloscope $(8 \mathrm{GHz})$. The DMD mirror array used in the experiment is protected by a cover glass which is AR coated for visible light. Under broadband and near infra-red (NIR) illumination, a strong interference results from the Fabry Perot effect between the DMD mirrors and the cover glass. This spectral interference transforms to a temporal modulation $(\sim 1.8 \mathrm{GHz})$ by dispersive time stretching. As a result, a low pass filter (LPF) with $1 \mathrm{GHz}$ cut-off is used after the detector to eliminate this undesired interference. In order to generate an arbitrary waveform, binary image patterns are created on the DMD chipset to set the states of micro mirrors accordingly to ensure the desired shape and the frequency. The binary image patterns similar to samples showed in Fig $2 \mathrm{a}$ are used to generate square and sawtooth waveforms, respectively. The unmodulated signal in which all the mirrors are assigned to ON state (white image pattern) and the modulated output signals are illustrated in Fig $2 \mathrm{~b}$.

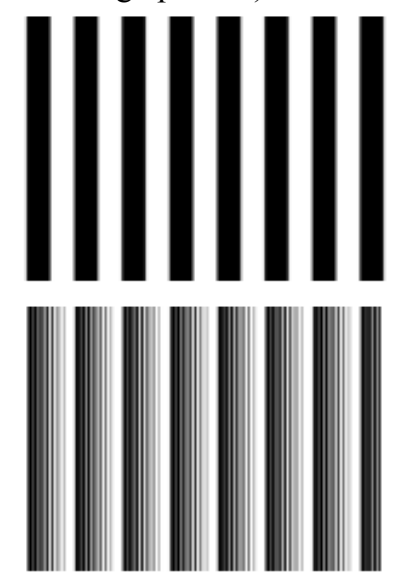

(a)
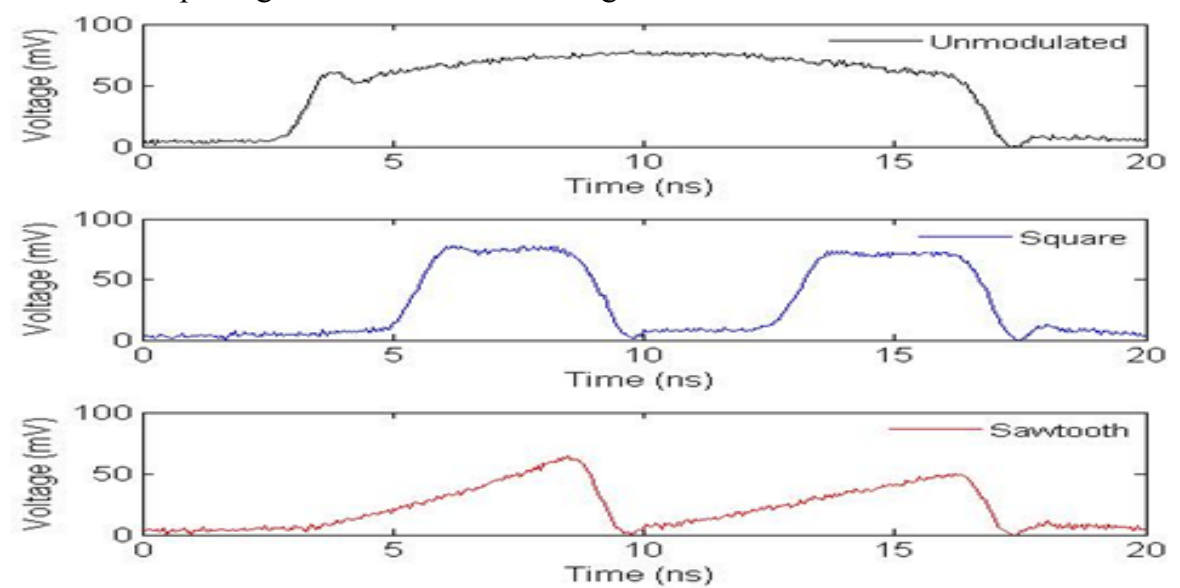

(b)

Fig 2. The sample binary image patterns (2a) created on DMD for square and sawtooth waveform generation. The detected unmodulated and the modulated square and sawtooth waveforms at $\sim 120 \mathrm{MHz}(2 \mathrm{~b})$.

As illustrated in Fig $2 b$, square and sawtooth waveforms at $\sim 120 \mathrm{MHz}$ are generated. The frequency of the RF waveform is determined by the spatial light modulator and the dispersive unit. By using low dispersion DCM and by increasing the spatial frequency of the modulation pattern, the frequency of the output waveform can be increased. On the other hand, the highest frequency (the resolution) that can be obtained in the system is mainly limited by the diffractive optics and the detection system. Using diffraction gratings with more groove density results in enhanced dispersion of the wavelengths over the entire DMD chipset. Since the diffraction limit determines the resolution of the space to wavelength mapping on DMD mirror array, reducing the focal size as well yield significant improvement in final resolution. In addition, by using a suitable cover glass which is AR coated for NIR illumination and using high speed detectors increases the temporal resolution and hence the smearing of the fine details of the arbitrary waveforms can be avoided.

\section{Conclusion}

We have introduced an optical AWG by using DMD mirror array as a spatial light modulator. By individually controlling the states of micro mirrors, square and sawtooth waveforms are generated to demonstrate the concept. Using an initial experimental setup, modulation rates up to $\sim 120 \mathrm{MHz}$ were observed at the output. By utilizing a state of the art MEMS technology, arbitrary waveforms with bandwidth $1 \mathrm{GHz}$ reconfigurable in $1 \mu$ s are achievable. The feedback control of the micro mirrors to eliminate the non-uniformity of the SC source and the system distortions is under investigation for generating high accuracy waveforms. Using an effective feedback algorithm, the system would converge to minimum achievable waveform error by compensating for the system non-idealities through the patterns fed into the mirrors.

\section{Acknowledgement}

This work is supported by DARPA Young Faculty Award, \#66001-10-1-4036, DARPA RADER Program and EU grant PIRG07-GA-2010-268370.

\section{References}

[1] L. Mullen, A. Vierina, P. Herczfeld, and V. Contarino, IEEE Trans. Microwave Theory Tech., Vol. 43, pp. 2370-77, Sept. 1995.

[2] A. M. Weiner, Prog. Quantum Electron, Vol. 19, pp. 1-237, 1995.

[3] Jason Chou, Yan Han, and Bahram Jalali, IEEE Photonics Technology Letters, Vol. 15, No. 4, April 2003.

[4] Mei-Li Hsieh, Optical Engineering 45, 3, March 2006.

[5] O. E. Martinez, IEEE J. Quantum Electron, Vol. QE-23, pp. 59-64, Jan. 1987. 